\title{
Statistical learnability of nuclear masses
}

\author{
A. Idini $\odot$ \\ Division of Mathematical Physics, Department of Physics, LTH, Lund University, \\ Post Office Box 118, S-22100 Lund, Sweden
}

(Received 9 April 2019; revised 22 July 2020; accepted 19 November 2020; published 14 December 2020)

\begin{abstract}
After more than 80 years from the seminal work of Weizsäcker and the liquid drop model of the atomic nucleus, deviations from experiments of mass models $(\sim \mathrm{MeV})$ are orders of magnitude larger than experimental errors $(\lesssim \mathrm{keV})$. Predicting the mass of atomic nuclei with precision is extremely challenging. This is due to the nontrivial many-body interplay of protons and neutrons in nuclei, and the complex nature of the nuclear strong force. Statistical theory of learning will be used to provide the bounds to prediction errors of a model trained with a finite data set. These bounds are validated with neural network models and compared with state of the art mass models. It will be argued that nuclear structure mass models explore a system on the limit of the precision bounds, as defined by the statistical theory of learning.
\end{abstract}

DOI: 10.1103/PhysRevResearch.2.043363

\section{INTRODUCTION}

Many relevant properties of atomic nuclei are extremely sensitive to their binding energy, i.e. mass, for example decay lifetimes and reaction rates. Therefore, there is the scientific need to reach the highest possible precision in models reproducing and predicting nuclear masses [1]. However, the current state of the art theoretical models deviate from experimental binding energies orders of magnitude more than the experimental errors. This article will investigate the difficulty of improving the precision of nuclear mass models from a statistical learning point of view.

The complexity of this problem was hinted in the context of chaotic quantum systems [2,3]. That is, the statistical distribution of masses shows a chaotic behavior that is formidable to deterministically reproduce (cf., also Ref. [4]). The amount of nuclear structure data available to study the mass distribution is related to the finite number of nuclear isotopes available in the laboratory and that can possibly exist. In particular, some mass models and density functional theory (DFT) access in principle only few properties of each nucleus, modeling only the ground state of the system. As of the last atomic mass evaluation (AME16), 3435 nuclei have been measured in the laboratories around the world [5]. Due to the lack of a comprehensive model of nuclear binding energies, it is not known how many nuclei could exist. The current consensus considers $\approx 7000$ nuclei [1]. In practice, many of these exotic nuclei will not be measured in the foreseeable future. The effect of the limited number of nuclear masses available in nature can be investigated using statistical learning theory.

Published by the American Physical Society under the terms of the Creative Commons Attribution 4.0 International license. Further distribution of this work must maintain attribution to the author(s) and the published article's title, journal citation, and DOI. Funded by Bibsam.
Statistical learning theory deals with the problem of devising a specific predictive model, belonging to a class of models $\Lambda$, using a set of data. When the number of data is finite, there is a limit to the precision that can be reached by a model. Qualitatively, the more a model is complex the more data it will need to reach a given predictive power. This paper will analyze the statistical learning bounds of deviation reachable by models attempting to reproduce and predict nuclear binding energies. This is done considering the mass model a statistical learning problem [6] with a complexity constrained by its parametric representation and the precision limited by the finite number of available training data points. Perceptron networks will be used to validate the statistical learning theory assumptions in this context. Finally, the bounds for notable density functionals will be examined. The tools here provided enable the analysis of the performance that can be expected from a model when the statistical treatment is rigorous and without bias.

\section{METHOD}

Statistical learning frames within quantifiable boundaries the effect that limited information has in the training of models [7]. The objective of a general learning problem is the minimization of the total risk functional $R(\alpha)$. That is, finding the set of parameters $\alpha$ within the model space $\Lambda$ by which a given model $f_{\alpha}$ best reproduces the data available and predicts the ones that could be taken under consideration. However, when working with a finite data set, what is actually minimized is the empirical risk $R_{e m p(l)}$. That is the risk evaluated over a finite number of training data $l$. Usually, the risk is defined using the root mean square deviation (RMSD) of model function or functional $f_{\alpha}$ with respect to the available data. Therefore, the value of $\alpha$ which minimizes $R_{\operatorname{emp}(l)}(\alpha)$ has to be found. This operation is often referred to as training. However, in principle, the minimization of the empirical risk (or training cost) does not guarantee the minimization of the total risk, that is the maximization of the predictive power of a theory. 
A model trained on limited data has only a probability of being generalizable keeping a good prediction error. In this case, the complexity of the model plays a role. Conventionally, a "simple" model generalizes better than a "complicated" one given similar performance on known data, because the complicated model is prone to overfitting. This is well known as the Occam's razor principle [8,9]. However, while these concepts are often considered in a qualitative way, statistical learning theory can quantify the tradeoff between complexity and data availability $[7,10,11]$.

First of all, the degree of complexity of a set of functions must be quantitatively defined. An established possibility is through the Vapnik and Chervonekis (VC) dimension [12]. The VC dimension is first defined for a set of Boolean functions $\Theta_{\alpha}(x)$, with $\alpha \in \Lambda$. The Boolean $\Theta_{\alpha}(x)$ can be either 0 or 1 for an input vector $x$. Given $h$ input vectors $x_{1}, \ldots, x_{h}$ there are at most $2^{h}$ possible results of $\Theta_{\alpha}\left(x_{i}\right)$ for $i=1, \ldots, h$. If $\Theta_{\alpha}$ has enough degrees of freedom to have $2^{h}$ possible results, varying the model parameters $\alpha$, it is said that $\Theta_{\alpha}$ shatters the set $x_{1}, \ldots, x_{h}$. The maximum number of input vectors $h$ that are shattered by $\Theta_{\alpha}$ is defined as the VC dimension. Therefore, the $\mathrm{VC}$ dimension is a measure of the effective degrees of freedom of a model.

The definition can be generalized for a bounded, real model $a \leqslant f_{\alpha}(x) \leqslant b$, with $a, b \in \mathbb{R}$, defining a corresponding set of Boolean functions,

$$
\Theta_{\alpha}(x, c)=\theta\left(f_{\alpha}(x)-c\right),
$$

with $\theta$ being the Heaviside unit step function $[\theta(z)=0$ for $z<0$, and $\theta(z)=1$ for $z \geqslant 0]$, and $c \in(a, b)$. The VC dimension of the set of real valued $f_{\alpha}(x)$ corresponds to the VC dimension of the set of the indicator functions $\Theta_{\alpha}(x, c)$ in Eq. (1) [13]. That is, the number of points in $\mathbb{R}$ the related indicator function (1) can shatter.

For example, a lower bound on the VC dimension of a polynomial class in $f: \mathbb{R}^{N} \rightarrow \mathbb{R}$ is given by lifting the polynomial to the space of its monomials. Each of the monomials will be able to shatter independently at least one vector because monomials are elements of a basis of polynomials. Therefore, the VC dimension is at least the number of monomials required to construct the polynomial, i.e. the number of coefficients [7].

Using VC dimension to quantify the data complexity of a model, it is possible to derive the minimum amount of data points needed to reach a total error $R(\alpha)$ with a certain probability. If a model class is bounded and has finite VC dimension, then it is possible to obtain a bound on the error that this model will have, with a given probability, with respect to the number of data provided. This paradigm is known as probably approximately correct (PAC) learning [14].

The lower bound of data points needed to learn a binary classifier up to a generalization error was demonstrated in Ref. [15]. The understanding of this case has been recently improved, demonstrating the exact bound [16]. The bound of the minimum number of examples needed to reach a given generalization error was demonstrated also for bounded functions in $\mathbb{Z}$ [17], and it has been extended to include noisy data [18]. The bound used in this work will be the simplest and general classifier bound and will be later applied to regression models.
To derive the bound of a regression model, let us consider the probability that a bad hypothesis $\Theta_{\text {bad }}$ will agree with a data point $x \rightarrow y$ is less than expected $1-\epsilon$, with $0<\epsilon<1$ the classification error. For $m$ independent training points, this implies $P\left[\Theta_{\text {bad }}\left(x_{1}\right)=y_{1}, \ldots, \Theta_{\text {bad }}\left(x_{m}\right)=y_{m}\right]<(1-\epsilon)^{m}<$ $e^{-m \epsilon}$. Considering the probability $\delta$ of a bad generalization, that is of agreeing with training and disagreeing with an external data point, $\delta \equiv P\left[\Theta_{\text {bad }}\left(x_{1}\right)=y_{1}, \ldots, \Theta_{\text {bad }}\left(x_{m}\right)=\right.$ $\left.y_{m}, \Theta_{\text {bad }}(\tilde{x}) \neq f(\tilde{x})\right]$, the union bound guarantees that this probability is

$$
\begin{aligned}
\delta<\sum_{\alpha \in \Lambda} P & {\left[\Theta_{\alpha}\left(x_{1}\right)=y_{1}, \ldots, \Theta_{\alpha}\left(x_{m}\right)=y_{m}, \Theta_{\alpha}(\tilde{x})=\tilde{y}\right] } \\
<|\Lambda| e^{-m \epsilon} &
\end{aligned}
$$

with $|\Lambda|$ being the dimension of the hypothesis space. In this way, it is possible to obtain the following bound on the number of data needed for a given accuracy,

$$
m \geqslant \frac{1}{\epsilon}\left[\ln (h)+\ln \left(\frac{1}{\delta}\right)\right],
$$

with $|\Lambda|$ taken as the VC dimension $h$ of the model class under consideration [6]. This procedure is known as empirical risk minimization and these are denoted as PAC learning bounds. It is of notice that there is no guarantee that the function $\Theta_{\alpha}$ with $\alpha \in \Lambda$ with error $\epsilon$ exists, but only a $1-\delta$ probability. Furthermore, this implies that is possible for the class of models under consideration to reproduce all data. Note also that this relation explains how it is possible to successfully train models that have a greater number of parameters than the data, a feature quite common in machine learning.

To apply the bound (3) for a mass model, it is convenient to consider the definition of the corresponding indicator function (1). The indicator function of a mass model will give the binding energy $E$ as a parameter, within the max-min interval $a=0 \mathrm{MeV}$ and $b=8.7945 \mathrm{MeV}$, which is the maximum binding energy per nucleon in the ${ }^{62} \mathrm{Ni}$ isotope. This interval can be normalized to $[0,1]$ for convenience. The indicator function will classify correctly if the predicted binding energy is correct. If the error is small and normally distributed, the error (normalized to 1) will represent the accuracy as in the derivation of (3). In the case of mass models, the error is indeed small compared to the binding energy, of the order of $1 \%$ or less. Therefore, using the indicator function it is possible to use the same definition of VC dimension $h$ and apply the derived bound (3) to estimate the number of data needed $m$ for a given precision $\epsilon$, assuming independent residuals.

To summarize within this context, a hypothesis space $\Lambda$ of mass models will be considered. In $\Lambda$, which complexity is characterized by the $\mathrm{VC}$ dimension $h$, there is a probability $1-\delta$ that a model exists so that the training error over $m$ data points is at most $\epsilon$ away from the total error over all nuclei, including the ones not yet discovered.

\section{NEURAL NETWORKS}

First, it is compelling to validate the assumptions used above to derive the PAC-learning bounds in this context. For this reason, several feed-forward neural networks with different properties have been trained. The data set used consists 


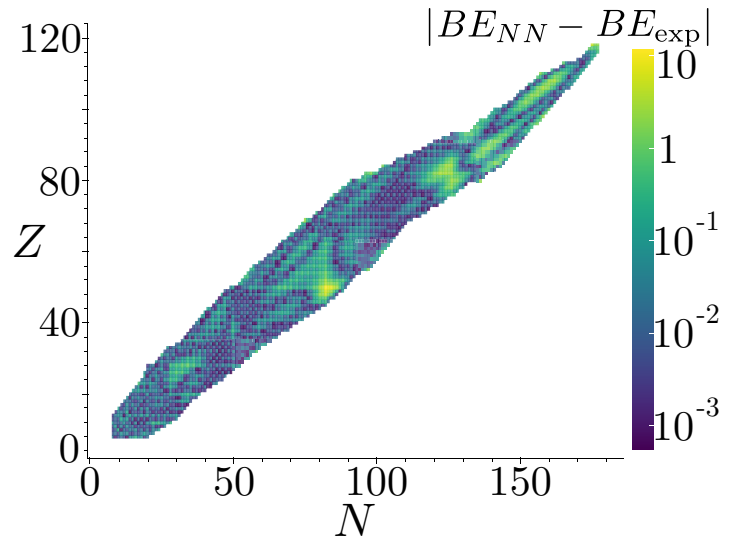

FIG. 1. Segre chart of the isotopes with the difference between the $N N 1$ neural network postdiction and experimental result in $\mathrm{MeV}$.

of the 2016 Atomic Mass Evaluation [5] (AME16), considering all nuclei with $N, Z \geqslant 8$, including the phenomenological estimates, for a total of 3336 nuclei and associated masses. The network must take $A, Z$ integer doublets and give back an $\mathbb{R}$ number, between 0 and 1 , to be converted to the binding energy $E$. Therefore, the network is a model $f_{\alpha}: \mathbb{N}^{2} \rightarrow \mathbb{R}$. $\alpha \in \Lambda$ are the parameters of the network. For this type of network, the VC dimension $h$ was demonstrated to be $O(N) \leqslant$ $h \leqslant O\left(N^{2}\right)[19,20]$, with $N$ being the number of weights.

The networks used are composed of an input layer with 50 sigmoid nodes, a single output node to give $E$, and a variable number of fully connected hidden layers $L$ of $n$ nodes to vary the $\mathrm{VC}$ dimension. The training uses a 0.5 dropout strategy, meaning that $50 \%$ of nodes in the hidden layers are randomly shut down, a procedure that inhibits overfitting [21]. This geometry of the neural network and training have been obtained as giving the most consistent and optimal results after a tree Parzen estimate hyperparameter optimization (based on expected improvement method [22]). The network setup and source code are available in the Supplemental Material [23]. Other than the sigmoid activation function, also rectified linear unit and softmax have been tried in all the possible combinations between input, hidden layers, and output. The resulting RMSD was not significantly impacted, testifying to the robustness of the PAC-learnable boundary. The number of weights for such a network is $51 n+n^{L}$, and therefore its $\mathrm{VC}$ dimension is at least $h \geqslant 51 n+n^{L}$. Of particular interest in this paper is the case of 1 hidden layer of 1000 nodes, denoted in the following as $N N 1$, selected as the example with $h \geqslant 52000$. Rectified linear unit in the hidden layer has been chosen for its piecewise linear structure that guarantees the neural network dimensionality bounds of Refs. [19,20].

The training loss is evaluated and optimized as RMSD between the calculated and experimental binding energies in a training set. The RMSD in Figs. 2 and 3 are evaluated on the validation set, using the $k$-fold cross-validation technique [24]. It consists of dividing the data set into $k$ training and validation exclusive subsets. The procedure is repeated several times with different separation of training and validation data set guaranteeing a bias-free assessment of the fitting procedure which improves on Bootstrap method [25]. Specifically, this method consists of the following:

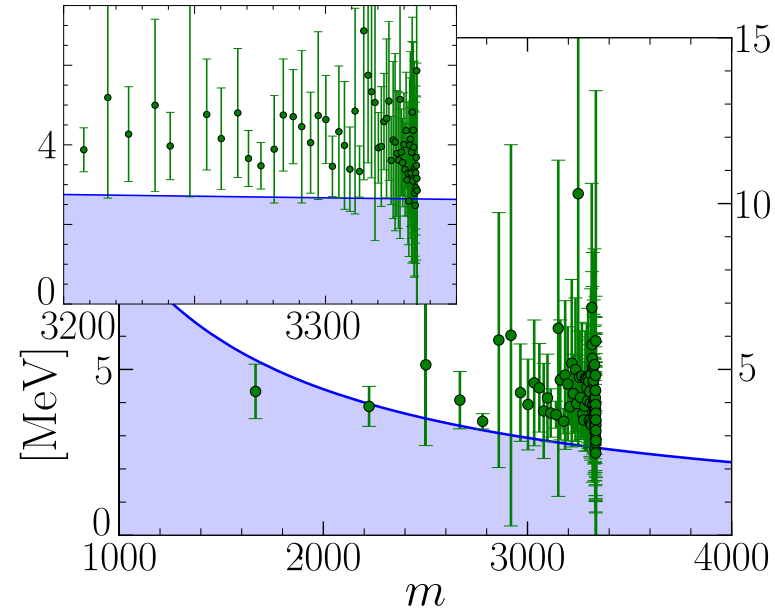

FIG. 2. Root mean square deviation of $N N 1$ on the $k$-fold cross validation of the AME16 data set (points). Related PAC-learning lower bound of the generalized error $\epsilon$ from (3) (line) and region forbidden by PAC learning bounds (shade) in function of the number of data $m$. Inset shows a closeup of the same figure on a region of interest.

(1) Divide the training set in a number of equivalent subsets $k$ (in this case randomly picked). This makes up the $k$ fold. A popular option, empirically verified to perform well in a variety of situations, is $k=10$.

(2) Train the set on a set composed of $k-1$ subsets; validate it on the remaining one.

(3) Repeat the training $k$ times, so that training and validation are considered over all the possible validation sets.

(4) From the RMSD resulting from the combination of training and validation, consider the average and the standard deviation of RMS deviations.

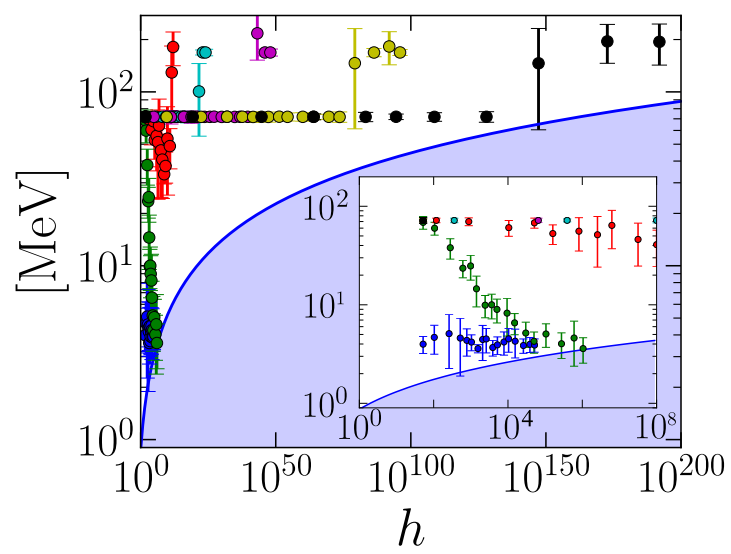

FIG. 3. Comparison of PAC lower bound of the error $\epsilon$ (3) and results for neural networks with different $\mathrm{VC}$ dimension $h$. Root mean square deviation of a feedforward neural network as described in the text on 10-fold cross validation of the AME16 data set (points). The number of hidden layers $L$ in figure are $L=1$ (green), $L=2$ (blue), $L=4$ (red), $L=8$ (cyan), $L=16$ (violet), $L=32$ (yellow), and $L=64$ (black). The number of nodes in each hidden layer then determines the VC dimension $h$. Lower bounds on $\epsilon$ from (3) and region forbidden by PAC learning bounds represented by line and shaded area respectively. Inset shows a closeup of the same figure on a region of interest. 
The average RMSD and its deviation will inform on the performance of the model and cost function chosen, and its resilience to modification of the data set and therefore predictive power. In this work, $k=10$ has been used. However, other $k$-fold choices were also tested, including a "complete crossvalidation," with no substantial difference to the distribution of RMSD.

To consider the potential of a pure neural network approach and this method of training and validating, it is interesting to investigate the best RMSD obtained for an example network. Neural network approaches to fit nuclear masses have been tried with specific configurations for neural network structure and propagation strategy [26,27], obtaining results between 0.7 and $5 \mathrm{MeV}$ of RMSD on masses on specific test sets. Note that the best results in Ref. [26] are obtained with the networks with the least number of weights, i.e. lower VC dimension. For the set of network considered, the best 10 -fold cross-validation instance on AME12 dataset [28] using $N N 1$, that is, 1000 rectified linear unit nodes in the hidden layer, was validated on AME16 obtaining $2.54 \mathrm{MeV}$ of RMSD, in Fig. 1. Furthermore, training directly on AME16, the average RMSD of the 10-fold total cross validation and the related deviation is $3.90 \pm 0.6 \mathrm{MeV}$. Considering instead the many combinations of $k$-fold cross validation represented in Fig. 2, representing an instance of complete cross validation, the distribution is $4.22 \pm 1.06 \mathrm{MeV}$.

Now it is possible to repeat the training procedure for several different neural networks varying the number of hidden layers and nodes. In this way, one can study if the PAC learning bounds hold for different orders of magnitude in the VC dimension in Fig. 3. The results presented in Figs. 2 and 3 testify to the reliability of PAC learning bounds for this system; the RMSD of models for different neuron number approaches the PAC bounds of $\epsilon$ when the network can be trained to reliably describe the system. This result is useful to relate the $\mathrm{VC}$ dimension of a mass model, the training data provided, with the total error. In the following, this result will be used to relate the VC complexity of known nuclear models with their expected performance.

\section{MASS MODELS AND DFT}

The Weizsäcker semiempirical mass formula [29,30] is one of the first attempts to globally describe the binding energy of atomic nuclei. It consists of a polynomial function of nucleon number $A$ and proton number $Z$,

$$
\begin{aligned}
E(A, Z)= & a_{v} A-a_{s} A^{2 / 3}-a_{C} \frac{Z(Z-1)}{A^{1 / 3}}-a_{a} \frac{(A-2 Z)^{2}}{A} \\
& +a_{p} \frac{\delta_{p}}{\sqrt{A}}
\end{aligned}
$$

where $\delta_{p}$ is 1 for $A$ and $Z$ even, 0 for $A$ or $Z$ odd, and -1 for $A$ and $Z$ odd. The coefficients of the semiempirical model mass formula consist of the well-known volume $a_{v}$, surface $a_{s}$, Coulomb $a_{C}$, asymmetry $a_{a}$, and pairing $a_{p}$ terms. The training was done over the AME12 database [28] and validated using the AME16 database [5]. The cost function
TABLE I. Coefficients in $\mathrm{MeV}$ of the semiempirical mass formula (4) obtained fitting AME12. Notice the uncertainties related to the different quantities, especially concerning pairing that testifies the softness of the regression of Weizsäcker mass formula respect to pairing, which is also the reason why different formulations are used for this term.

$$
\begin{aligned}
& a_{v}=15.40 \pm 0.014 \\
& a_{s}=16.71 \pm 0.042 \\
& a_{C}=0.701 \pm 0.001 \\
& a_{a}=22.56 \pm 0.037 \\
& a_{p}=11.88 \pm 0.823
\end{aligned}
$$

adopted is the modified $\chi^{2}$,

$$
\tilde{\chi}^{2}=\frac{\sum_{i}\left(f_{\alpha}\left(x_{i}\right)-y_{i}\right)^{2}}{\left[\ln \left(\Delta y_{i}\right)\right]^{2}},
$$

that has been adjusted in the case measured data $y_{i}$ have errors $\Delta y_{i}$ that span different orders of magnitude, as for the case of errors in mass measurements, from Ref. [31]. The related uncertainties have been computed with the covariance matrix calculated as inverse of the Hessian, which is composed of derivatives of the cost function respect to the free parameters. By linear approximation, it is possible to estimate errors related to a parameter or observable [31-33]. The obtained parameters and related uncertainties are reported in Table I. To be noted that the results are similar to those of Ref. [34] (obtained independently and with different fitting procedures).

To calculate the VC dimension of the semiempirical mass formula, it is straightforward to consider this notable polynomial,

$$
E(N, Z)=\sum_{i, j=0}^{N} a_{i j} A^{i} Z^{j},
$$

where in mass models $A$ is the total number of nucleons, $Z$ is the atomic charge or number of protons, and the binding energy $E$ is parametrized as a polynomial of these variables. As stated in the previous section, the VC dimension is the number of coefficients. For this polynomial, it is $h \geqslant(N+1)^{2}$. Therefore, it is possible to relate the semiempirical mass formula to a $\mathrm{VC}$ dimension of at least $[(N=6)+1]^{2}$, considering with no bias all the possible combinations of radius, surface, volume, and symmetry terms, or a VC dimension of 6 , considering the traditional parametrization derived with the physical bias.

Density functional theory is used to describe systems composed of many quantum particles [35] and has been an extremely successful model of atomic nuclei [36,37]. It is based on the Hohenberg-Kohn theorems [38]: (i) Ground-state properties of a many-fermion system are uniquely determined by the fermion density and (ii) there exists one and only one density that minimizes the functional; that is, the groundstate density and its energy density functional returns the ground-state energy. This latter preposition is known as the variational principle. In other words, the model functional of density $\rho(x), E[\rho(x)]$, will be minimized varying the densities with some Lagrangian constraints (e.g., that the densities contain the correct number of particles) and its minimum will 
correspond to the exact ground-state density and corresponding energy.

The functional $E_{\alpha}[\rho(x)]$ is usually a complicated combination of densities, eventually derived from a pseudopotential or functional generator $[33,39]$. The parameters $\alpha$ of the pseudopotential are tuned to reproduce physical ground-state properties. The densities and properties are calculated through the variational principle. Therefore, the same principle of risk minimization and consequent bounds applies. A conservative lower bound to the VC dimension of functionals can be provided considering that

$$
h(E[\rho(x)]) \leqslant h(E(x)),
$$

that in turn determines a lower PAC bound to the error and number of data points.

In nuclear physics, several functional forms and hundreds of parametrizations have been advanced in the DFT spirit. In recent times, many new approaches have been tried considering high-order expansions $[33,40]$. One of the most successful functional forms is the Skyrme density functional. This form is made of a momentum expansion of contact terms, that is, a combination of Dirac $\delta$ with momentum-dependent terms (which translates in derivatives of the densities in the functional), ${ }^{1}$ density-dependent, and spin-orbit terms.

The functional can be related to a polynomial expansion of the density (excluding additional terms) using (7), reducing to the VC complexity of a second-order polynomial ${ }^{2}$ over two dimensions (neutrons and protons) and eight constraints on the parameters, therefore with dimension at least $h_{\text {Skyrme }} \geqslant$ $2[(N=2)+1]^{2}-8=10$. This estimate agrees with the 10 free parameters of a first-order Skyrme functional. However, it is not always the case that the VC dimension corresponds to the number of parameters. As a title of example, in the case of the Gogny functional [41] the pseudopotential is composed of two Gaussians with different widths and a density-dependent term. The Gaussian itself has VC dimension 3 , because its classifier can shatter three points. There are four terms for each Gaussian, plus the additional densitydependent and spin-orbit terms, and hence $h_{\text {Gogny }} \geqslant 14$. New generation functionals are increasing the number of parameters and considering higher order terms. For example, the $\mathrm{N}^{3}$ LO Reg6d [42] has 16 parameters on Gaussians, plus the spin-orbit and density-dependent term. Therefore, $h_{\text {Reg6d }} \geqslant$ $16 * 3+2=50$.

\section{DISCUSSION AND CONCLUSIONS}

Neural network models have been recently used to reproduce Hartree Fock Bogoliubov calculations [45] and other nuclear properties, such as radii [46]. Neural networks have also recently emerged as a possible method for providing additional corrections and correlations (arguably representing

\footnotetext{
${ }^{1}$ Interestingly, Theorem 6.8 and the following in Ref. [7] state that a good rate of convergence can be reached only for smooth functions. Despite being derived from pseudopotentials with difficult discontinuities, the resulting densities are smooth and therefore arguably can be converged.

${ }^{2}$ First-order derivatives go to 1 , and other terms cancel.
}

TABLE II. Properties of different models to describe nuclear physics masses. The columns represent (i) the lower bound on VC dimension for the given model; (ii) RMSD of the referred models; (iii) lower bound on the $\epsilon$ error provided by PAC learning considering 3336 homogeneously weighted data points; and (iv) number of data points $\tilde{m}$ needed to reach an error $\epsilon$ of $100 \mathrm{keV}$ with $99 \%$ probability, a considerable improvement to current bounds. Note that the RMSD result for Gogny D1M in Ref. [44] contains beyond-DFT corrections. RMSD for $N N 1$ is obtained by averaging all the results in Fig. 2.

\begin{tabular}{lcccc}
\hline \hline Model & VC-dim & RMSD [MeV] & $\epsilon[\mathrm{MeV}]$ & $\tilde{m}$ \\
\hline NN1 & 52000 & $4.22 \pm 1.06$ & 2.64 & 88076 \\
Weizsäcker & 6 & $3.41 \pm 0.19$ & 1.09 & 36434 \\
& 49 & & 1.45 & 48395 \\
Skyrme (UNEDF0) & 10 & $1.428[43]$ & 1.18 & 39343 \\
Gogny (D1M)* & 14 & $0.798[44]$ & 1.24 & 41259 \\
N $^{3}$ LO Reg6d & 50 & $1.46[42]$ & 1.45 & 48510 \\
\hline \hline
\end{tabular}

missing shell effects) to a previously devised mass model with excellent results $[47,48]$. This work provides additional information and an interpretation of the relative success neural network models have in modeling ground-state properties in nuclear physics.

Most importantly, a long-standing problem in the creation of nuclear density functionals and mass models is the number and type of data that has to be included in their fitting. This is particularly important in the study of next-generation, high-order, nuclear density functionals [33,40,42]. This work moves toward quantifying the amount of data needed to reach an expected precision of a given mass model. Table II shows a comparison of known mass models RMSD and PAC-learning bounds. The interpretation of Table II comes with several caveats.

The RMSD related to Skyrme UNEDF0 and Gogny D1M are calculated on the data available in the atomic mass evaluation 2003 [49]. Moreover, in the case of Skyrme UNEDF0 only even-even nuclei are considered, while for REG6d only a subset of 214 spherical even-even nuclei is. By definition, a RMSD on a limited amount of data is the empirical risk and cannot be considered a total risk or generalization error, even more so when calculated on data belonging to the training set. However, one can safely assume that the total error will not be better than the empirical risk. Most importantly, the objective of such functionals is to reproduce not only masses but other properties as well, optimizing a complicated cost function. Physical bias enters in the construction of this cost function, even when the statistical approach is rigorous, such as in the UNEDF program [50] and to a greater extent in the definition of other successful models. Sometimes functionals have been developed as interactions, considering beyond mean field correlations in the total energy and other observables; e.g., Gogny pairing was tuned to scattering data. Furthermore, the RSMD value cited for Gogny D1M includes beyond DFT physics and additional corrections. This testifies to the success of including more observables and many-body correlations. Note also that parameters can be redundant or not sensitive to the observables under consideration $[33,34,50]$. This, in principle, decreases the $\mathrm{VC}$ dimensionality with respect to the observable under consideration, but also the post- and predictive power of the model. 
Despite these caveats, the close values of the bound $\epsilon$, the neural network calibration, and the RMSD of state of the art models suggest the possibility discussed herein of investigating the limits of precision in mass models through statistical learning as defined by PAC learning. An important conclusion for the development of functionals is that the training complexity is only logarithmically dependent on the $\mathrm{VC}$ dimension. Therefore, higher order functionals can be developed with excellent performance with only a modest increase in the number of data. However, the number of data needed for a significant improvement of all models under consideration is of the order of $\tilde{m} \gtrsim 4 \times 10^{4}$ according to PAC learning bounds. The relation between $\epsilon$ and RMSD appears robust to the addition of a few more data points; hence, many more data and observables need to be considered to reach such an improvement, either explicitly or implicitly through training bias.

This work is just a first step in the evaluation of statistical learning precision bounds in the description of many-body systems. Further investigations on different models, more complete properties, and other statistical learning prescriptions will be required in the future. In particular, agnostic learning can provide further information on the best possible model present in a given model class. Support vector machines obtained interesting results of accuracy and were investigated for nuclear mass predictions with good results [51]. These would also be an interesting object of study in light of PAC learning, considering the well-established relations between the two methods [7].

However, some conclusions can be drawn with better hindsight than previously possible. In light of this work, the difficulty in developing higher order, higher complexity func- tionals and mass models to reach a predictive and precise estimate of nuclear masses might not be a shortcoming of some specific model. Instead, the necessary information for a model to predict with the desired degree of precision is larger than masses (or few ground-state properties) alone. The number of data included in the learning of most nuclear EDF models is presently insufficient for a leap in precision. To substantially increase the performance of these models, especially in biasless, statistically robust, next generation functionals, a variety of observables must be included. Eventually, investigating the response to fields will be crucial. This will involve $a b$ initio calculations (that is, interaction including nucleon-nucleon scattering data) or unification of structure and reactions.

This work has investigated the VC dimension related to a many-body method and its implication regarding the performance that a given model and training can reach. This work suggests that many-body methods might not only be judged by their computational complexity, as in the novel field of Hamiltonian complexity [52,53], but also in terms of their information complexity represented by $\mathrm{VC}$ dimension and PAC learning bounds.

\section{ACKNOWLEDGMENTS}

This work benefited from discussions with the participants to the workshop "Novel approaches for the description of heavy nuclei" organized at Lund University 19-21 March 2019, with the contribution of Newton Alumni Fellowship of the Royal Society. The Quadro P6000 GPU used for this research was donated by the NVIDIA Corporation. The source code used is available in the Supplemental Material [23].
[1] W. Nazarewicz, The limits of nuclear mass and charge, Nat. Phys. 14, 537 (2018).

[2] S. Åberg, Nuclear physics: Weighing up nuclear masses, Nature (London) 417, 499 (2002).

[3] O. Bohigas and P. Leboeuf, Nuclear Masses: Evidence of OrderChaos Coexistence, Phys. Rev. Lett. 88, 092502 (2002).

[4] J. Barea, A. Frank, J. G. Hirsch, and P. Van Isacker, Nuclear Masses Set Bounds on Quantum Chaos, Phys. Rev. Lett. 94, 102501 (2005).

[5] M. Wang, G. Audi, F. Kondev, W. Huang, S. Naimi, and X. Xu, The AME2016 atomic mass evaluation (II): Tables, graphs, and references, Chin. Phys. C 41, 030003 (2017).

[6] V. N. Vapnik, An overview of statistical learning theory, IEEE Trans. Neural Networks 10, 988 (1999).

[7] V. N. Vapnik, The Nature of Statistical Learning Theory (Springer-Verlag, Berlin, 1995).

[8] A. Blumer, A. Ehrenfeucht, D. Haussler, and M. K. Warmuth, Occam's razor, Inf. Process. Lett. 24, 377 (1987).

[9] P. Domingos, The role of Occam's razor in knowledge discovery, Data Mining Knowl. Disc. 3, 409 (1999).

[10] V. Vapnik, Principles of risk minimization for learning theory, in NIPS'91: Proceedings of the 4th International Conference on Neural Information Processing Systems (Morgan Kaufmann Publishers Inc., San Francisco, CA, 1991), pp. 831-838.
[11] J. Shawe-Taylor, P. L. Bartlett, R. C. Williamson, and M. Anthony, Structural risk minimization over data-dependent hierarchies, IEEE Trans. Inf. Theory 44, 1926 (1998).

[12] V. Vapnik and A. Chervonenkis, On the uniform convergence of relative frequencies of events to their probabilities, Theory Probab. Appl. 16, 264 (1971).

[13] T. Hastie, R. Tibshirani, and J. Friedman, The Elements of Statistical Learning: Data Mining, Inference, and Prediction, 2nd ed., Springer Series in Statistics (Springer, New York, 2009).

[14] L. G. Valiant, A theory of the learnable, Commun. ACM 27, 1134 (1984).

[15] A. Ehrenfeucht, D. Haussler, M. Kearns, and L. Valiant, A general lower bound on the number of examples needed for learning, Inf. Comput. 82, 247 (1989).

[16] A. Kontorovich and I. Pinelis, Exact lower bounds for the agnostic probably-approximately-correct (PAC) machine learning model, Ann. Statist. 47, 2822 (2019).

[17] D. Haussler, Decision theoretic generalizations of the PAC model for neural net and other learning applications, Inf Comput. 100, 78 (1992).

[18] P. L. Bartlett, P. M. Long, and R. C. Williamson, Fat-shattering and the learnability of real-valued functions, J. Comput. Syst. Sci. 52, 434 (1996). 
[19] W. Maass, Vapnik-Chervonenkis dimension of neural nets, in Handbook of Brain Theory and Neural Networks, edited by M. A. Arbib (MIT Press, Cambridge, MA, 1995), pp. 10001003.

[20] P. Koiran and E. D. Sontag, Neural networks with quadratic VC dimension, J. Comput. Syst. Sci. 54, 190 (1997).

[21] N. Srivastava, G. Hinton, A. Krizhevsky, I. Sutskever, and R. Salakhutdinov, Dropout: A simple way to prevent neural networks from overfitting, J. Mach. Learn. Res. 15, 1929 (2014).

[22] M. Shelley, P. Becker, A. Gration, and A. Pastore, Advanced statistical methods to fit nuclear models, Acta Phys. Polon. B Proc. Suppl. 12, 649 (2019).

[23] See Supplemental Material at http://link.aps.org/supplemental/ 10.1103/PhysRevResearch.2.043363 for the source code used consisting of the neural network training and calculation of PAC-learning bounds.

[24] P. A. Lachenbruch and M. R. Mickey, Estimation of error rates in discriminant analysis, Technometrics 10, 1 (1968).

[25] B. Efron, Estimating the error rate of a prediction rule: Improvement on cross-validation, J. Am. Stat. Assoc. 78, 316 (1983).

[26] K. Gernoth, J. Clark, J. Prater, and H. Bohr, Neural network models of nuclear systematics, Phys. Lett. B 300, 1 (1993).

[27] S. Athanassopoulos, E. Mavrommatis, K. Gernoth, and J. Clark, Nuclear mass systematics using neural networks, Nucl. Phys. A 743, 222 (2004).

[28] G. Audi, F. G. Kondev, M. Wang, B. Pfeiffer, X. Sun, J. Blachot, and M. MacCormick, The NUBASE2012 evaluation of nuclear properties, Chin. Phys. C 36, 1157 (2012).

[29] C. F. v. Weizsäcker, Zur Theorie der Kernmassen, Z. Phys. 96, 431 (1935).

[30] H. A. Bethe and R. F. Bacher, Nuclear physics A: Stationary states of nuclei, Rev. Mod. Phys. 8, 82 (1936).

[31] J. Dobaczewski, W. Nazarewicz, and P.-G. Reinhard, Error estimates of theoretical models: A guide, J. Phys. G: Nucl. Part. Phys. 41, 074001 (2014).

[32] J. Toivanen, J. Dobaczewski, M. Kortelainen, and K. Mizuyama, Error analysis of nuclear mass fits, Phys. Rev. C 78, 034306 (2008).

[33] K. Bennaceur, A. Idini, J. Dobaczewski, P. Dobaczewski, M. Kortelainen, and F. Raimondi, Nonlocal energy density functionals for pairing and beyond-mean-field calculations, J. Phys. G: Nucl. Part. Phys. 44, 045106 (2017).

[34] A. Pastore, An introduction to bootstrap for nuclear physics, J. Phys. G: Nucl. Part. Phys. 46, 052001 (2019).

[35] D. S. Sholl and J. A. Steckel, Density Functional Theory: A Practical Introduction (John Wiley and Sons, New York, 2009).

[36] M. Bender, P.-H. Heenen, and P.-G. Reinhard, Self-consistent mean-field models for nuclear structure, Rev. Mod. Phys. 75, 121 (2003).
[37] P. Ring and P. Schuck, The Nuclear Many-Body Problem (Springer, Berlin, 1980).

[38] P. Hohenberg and W. Kohn, Inhomogeneous electron gas, Phys. Rev. 136, B864 (1964).

[39] E. Perlińska, S. G. Rohoziński, J. Dobaczewski, and W. Nazarewicz, Local density approximation for proton-neutron pairing correlations: Formalism, Phys. Rev. C 69, 014316 (2004).

[40] D. Davesne, A. Pastore, and J. Navarro, Skyrme effective pseudopotential up to the next-to-next-to-leading order, J. Phys. G: Nucl. Part. Phys. 40, 095104 (2013).

[41] J. Dechargé and D. Gogny, Hartree-Fock-Bogolyubov calculations with the $D 1$ effective interaction on spherical nuclei, Phys. Rev. C 21, 1568 (1980).

[42] K. Bennaceur, J. Dobaczewski, T. Haverinen, and M. Kortelainen, Properties of spherical and deformed nuclei using regularized pseudopotentials in nuclear DFT, J. Phys. G: Nucl. Part. Phys. 47, 105101 (2020).

[43] M. Kortelainen, J. McDonnell, W. Nazarewicz, E. Olsen, P.-G. Reinhard, J. Sarich, N. Schunck, S. M. Wild, D. Davesne, J. Erler, and A. Pastore, Nuclear energy density optimization: Shell structure, Phys. Rev. C 89, 054314 (2014).

[44] S. Goriely, S. Hilaire, M. Girod, and S. Péru, First GognyHartree-Fock-Bogoliubov Nuclear Mass Model, Phys. Rev. Lett. 102, 242501 (2009).

[45] T. Bayram, S. Akkoyun, and S. O. Kara, A study on groundstate energies of nuclei by using neural networks, Ann. Nucl. Energy 63, 172 (2014).

[46] S. Akkoyun, T. Bayram, S. O. Kara, and A. Sinan, An artificial neural network application on nuclear charge radii, J. Phys. G: Nucl. Part. Phys. 40, 055106 (2013).

[47] R. Utama and J. Piekarewicz, Refining mass formulas for astrophysical applications: A Bayesian neural network approach, Phys. Rev. C 96, 044308 (2017).

[48] Z. Niu and H. Liang, Nuclear mass predictions based on Bayesian neural network approach with pairing and shell effects, Phys. Lett. B 778, 48 (2018).

[49] A. Wapstra, G. Audi, and C. Thibault, The AME2003 atomic mass evaluation, Nucl. Phys. A 729, 129 (2003).

[50] M. Kortelainen, T. Lesinski, J. Moré, W. Nazarewicz, J. Sarich, N. Schunck, M. V. Stoitsov, and S. Wild, Nuclear energy density optimization, Phys. Rev. C 82, 024313 (2010).

[51] H. Li, J. W. Clark, E. Mavrommatis, S. Athanassopoulos, and K. A. Gernoth, Modeling nuclear properties with support vector machines, arXiv:nucl-th/0506080.

[52] J. D. Whitfield, P. J. Love, and A. Aspuru-Guzik, Computational complexity in electronic structure, Phys. Chem. Chem. Phys. 15, 397 (2013).

[53] S. Gharibian, Y. Huang, Z. Landau, and S. W. Shin, Quantum Hamiltonian complexity, Found. Trends Theor. Comp. Sci. 10, 159 (2015). 